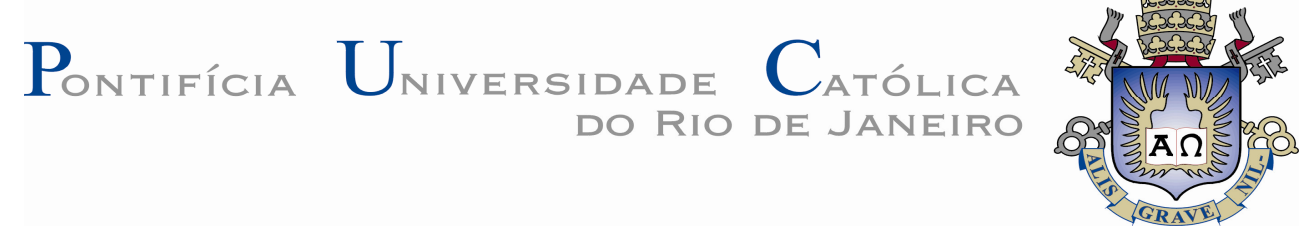

Renato Marçullo Borges

\title{
Determinação de Sn em leite humano por espectrometria de absorção atômica no forno de grafite.
}

\section{Dissertação de Mestrado}

Dissertação apresentada como requisito parcial para obtenção do título de Mestre pelo Programa de Pós-Graduação em Química da PUC-Rio.

Orientadores: Prof. Reinaldo Calixto de Campos Dra. Maria de Fátima Ramos Moreira 


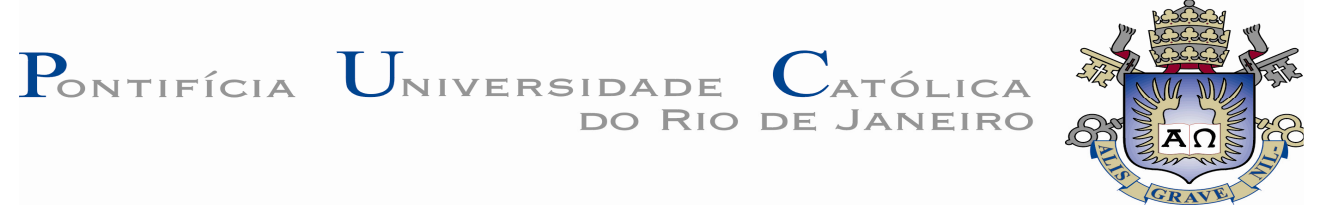

Renato Marçullo Borges

\section{Determinação de Sn em leite humano por espectrometria de absorção atômica no forno de grafite.}

Dissertação apresentada como requisito parcial para obtenção do título de Mestre pelo Programa de PósGraduação em Química da PUC-Rio. Aprovada pela Comissão Examinadora abaixo assinada.

Prof. Reinaldo Calixto de Campos

Orientador

Departamento de Química - PUC-Rio

Dra. Maria de Fátima Ramos Moreira

Co-orientador

Co-orientadora - CESTEH/ENSP/FIOCRUZ

Prof. Marco Aurelio Zezzi Arruda Instituto de Química - UNICAMP

Prof. Olaf Malm

Instituto de Biofísica - UFRJ

Prof. José Eugenio Leal

Coordenador Setorial do Centro Técnico Científico -PUC-Rio

Rio de Janeiro, 28 de agosto de 2008. 
Todos os direitos reservados. É proibida a reprodução total ou parcial do trabalho sem autorização da universidade, da autora e do orientador.

\section{Renato Marçullo Borges}

Graduou-se em bacharel e licenciatura em química pela Universidade do Grande Rio (2005). Atualmente é funcionário da Fundação Oswaldo Cruz.

Ficha Catalográfica

Borges, Renato Marçullo

Determinação de Sn em leite humano por espectrometria de absorção atômica eletrotérmica / Renato Marçullo Borges ; orientadores: Reinaldo Calixto de Campos, Maria de Fátima Ramos Moreira. -2008.

82 f. : il.(color.) ; $30 \mathrm{~cm}$

Dissertação (Mestrado em Química)-Pontifícia Universidade Católica do Rio de Janeiro, Rio de Janeiro, 2008.

Inclui bibliografia

1. Química - Teses. 2. Estanho. 3. Leite humano. 4. GFAAS. 5. Determinação direta. I. Campos, Reinaldo Calixto de. II. Moreira, Maria de Fátima Ramos. III. Pontifícia Universidade Católica do Rio de Janeiro. Departamento de Química. IV. Título. 
Primeiramente à Deus, que sem Sua permissão não seria possível chegar até aqui. E aos meus pais, Vera e Adérito, por mostrarem o caminho da simplicidade, do amor e do trabalho 


\section{Agradecimentos}

Ao professor Reinaldo Calixto de Campos por seu apoio e orientação.

A $\operatorname{Dr}^{\mathrm{a}}$ Maria da Fátima Ramos Moreira que foi a grande incentivadora, professora e amiga que me acolheu em seu laboratório desde os tempos de ingênuo aluno de graduação.

Ao professores Odir Clécio, Rosália Maria, Juremí Carvalho e Dalton Marcondes por permitirem e apoiarem meu ingresso no mestrado.

Aos professores da banca examinadora.

Ao meus irmãos Paula e Eduardo, sempre presentes em todos os momentos da minha vida.

Aos meus amigos, Vítor Hugo, Leonardo, Bárbara, Cristiano, Monique, Cíntia, Evandro, Leandro e Thaísa pelo apoio e compreensão nos momentos de ausência devido a elaboração desta dissertação.

A Renata Spolti Leão, que com sua intervenção permitiu a continuação deste trabalho e pela constante preocupação.

As minhas colegas de mestrado, Thaísa, Cabrini, Ana Paula e Amanda, sem 
vocês essa etapa seria mais difícil.

A Maria de Fátima da Silva Almeida, por ser meu anjo de guarda nos prazos, inscrições, notas e acima de tudo por ser minha amiga, meus parabéns!

Ao pessoal do laboratório Regina, Fernanda, Aline, Sayonara, Beatriz, Débora, Mônica e Sandra pelo apoio e carinho dispensados no dia-a-dia.. 


\section{Resumo}

Borges, Renato Marçullo; Campos, Reinaldo Calixto de; Moreira, Maria de Fátima Ramos, Determinação de Sn em leite humano por espectrometria de absorção atômica no forno de grafite, Rio de Janeiro, 2008, 82p, Dissertação de Mestrado - Departamento de Química, Pontifícia Universidade Católica do Rio de Janeiro.

O estanho é um metal de ocorrência natural que é extraído do minério de cassiterita na forma de $\mathrm{SnO}_{2}$. É lançado na natureza através de fontes naturais e antrópicas e ocorre na natureza tanto na forma inorgânica, quanto orgânica. Quanto menor a cadeia orgânica associada ao metal, maior a toxicidade do composto. Compostos orgânicos podem penetrar na membrana celular e interromper a fosforilação oxidativa e danificar as mitocôndrias. Podem ser ainda genotóxicos e imunotóxicos. O leite humano é a principal fonte de alimentação e nutrição para recém-nascidos, e é encorajada até os 2 anos de idade. Entretanto, é também a principal fonte de exposição de substâncias exógenas para bebês, incluindo o estanho e seus compostos, já que pode ser a rota de excreção de poluentes ambientais oriundos da mãe. A determinação direta do leite por EAA-FG é interessante do ponto de vista analítico pois reduz o tempo de preparação e a possibilidade de contaminação da amostra. Neste trabalho, foi utilizado um forno de grafite com aquecimento transversal. Curvas de pirólise e atomização levaram a temperaturas de pirólise e atomização de 1300 e $2200^{\circ} \mathrm{C}$, respectivamente, usando um volume de amostra de $20 \mu \mathrm{L}$. Os melhores resultados foram obtidos com a diluição $1+1$ em $\mathrm{HNO}_{3} \quad 0,2 \%$ v/v e com a massa de $10+5 \mu \mathrm{g}$ de paládio+magnésio, para um volume de $10 \mu \mathrm{L}$. Em todos os casos, a atenuação de fundo foi adequada para o corretor baseado no efeito Zeeman. A curva analítica foi linear até $300 \mu \mathrm{g} . \mathrm{L}^{-1}$, tanto no meio aquoso quanto na presença da matriz, entretanto a calibração teve que ser realizada com a adição de soluções de calibração à matriz. $O$ limite de detecção $(n=10, k=3)$ foi 
de 0,6 $\mu \mathrm{g} \mathrm{Sn.L^{-1 }}$ na amostra original. Foi verificado que a presença de $\mathrm{Cl}^{-1} \mathrm{e} \mathrm{Ca}^{+2}$ em concentrações acima de 100 e $40 \mathrm{mg} . \mathrm{L}^{-1}$, respectivamente, depreciam fortemente o sinal de estanho. Por outro lado, o aumento do sinal de estanho ocorre devido a presença de $\mathrm{P}$ em concentrações que variam entre 5 e $150 \mathrm{mg} . \mathrm{L}^{-}$ ${ }^{1}$. Recuperações maiores que $95 \%$ de iSn no leite humano foram obtidas como parte do estudo de calibração. A metodologia desenvolvida provou ser adequada para a determinação de $\mathrm{Sn}$, quando na forma de MMT, através da calibração de iSn como analito de calibração..

\section{Palavras-chave}

Estanho, leite humano. GFAAS, determinação direta. 


\section{Abstract}

Borges, Renato Marçullo; Campos, Reinaldo Calixto de(Advisor); Moreira, Maria de Fátima Ramos, Determination of Sn in human milk by graphite furnace atomic absorption spectrometry, Rio de Janeiro, 2008, 82p, MSc. Dissertation - Departamento de Química, Pontifícia Universidade Católica do Rio de Janeiro..

Tin is a metal of natural occurrence which is mainly extracted from the mineral cassiterite, $\mathrm{SnO}_{2}$. Tin may be released to the atmosphere from both natural and anthropogenic sources. In nature, it occurs in both inorganic and organic forms, and the shorter the organic chain associated to the metal, the higher the toxicity of the compound. Organotin compounds can penetrate cell membranes and cause damage to cell, interrupt oxidative phosphorylation and damage mitochondria. They can be immunotoxic and genotoxic. Human milk is the ideal food for newborns due to its composition and availability. Human milk is the fundamental food for infants, thus breast feeding is greatly encouraged up to 2 years. However, it is also the major source of exposition to exogenous substances for newborns, including tin and its compounds, since breast milk can also be a route of maternal excretion of undesired environmental pollutants. The direct GF AAS analysis of products such as milk is very attractive, due to the inherent simplicity, and decrease in the sample preparation time and contamination chances. In the present work, a transversally heated graphite atomizer was used. Pyrolysis and atomization temperatures curves led to pyrolysis and atomization temperatures of 1300 and $2200^{\circ} \mathrm{C}$, respectively, using a sample volume of $20 \mu \mathrm{L}$. The use of a $1+1$ dilution factor using $0.2 \% \mathrm{v} / \mathrm{v}$ $\mathrm{HNO}_{3}$ as diluent showed to be the best choice, as well as a modifier mass of $10+5 \mu \mathrm{g}$ of palladium+magnesium, respectively. The modifier solution volume was $10 \mu \mathrm{L}$. In all cases, the background attenuation was well within the limits of the Zeeman effect based background corrector. The analytical curve was linear 
up to $300 \mu \mathrm{g} \mathrm{L}^{-1}$, in both aqueous medium as well as in the presence of the matrix. Calibration had to be performed with matrix matched calibration solutions, using a blank level sample. The limit of detection $(n=10, k=3)$ in the original sample was $0.6 \mu \mathrm{g} \mathrm{L}^{-1}$. It was verified that $\mathrm{Cl}^{-1}$ and $\mathrm{Ca}^{2+}$ influence on tin absorbance signal is markedly depreciated at concentrations above 100 and 40 $\mathrm{mgL}^{-1}$, respectively. On the other hand, the increase of tin signal is caused by the presence of $\mathrm{P}$ in concentrations between 5 and $150 \mathrm{mg} . \mathrm{L}^{-1}$. In a calibration study, recovery was higher than $95 \%$ in human milk. The methodology was able in determine $\mathrm{Sn}$, as MMT, when iSn is used for the calibration.

\section{Keywords}

Tin, Human milk, GFAAS, direct determination. 


\section{Sumário}

$\begin{array}{ll}1 \text { Introdução } & 18\end{array}$

$\begin{array}{ll}2 \text { Objetivo } & 21\end{array}$

3 Produção, usos e aplicações do estanho 22

4 Formas de contaminação (risco à saúde). 25

4.1. Toxicocinética. 28

4.1.1. Metabolismo. 31

4.1.2. Eliminação e excreção 31

4.1.3. Efeitos na saúde humana (toxicodinâmica) 32

5. Leite humano. 36

6. Métodos analíticos para a determinação de estanho 39

7. Experimental 44

7.1. Instrumental 44

7.2. Reagentes, soluções e materiais 45

7.3. Armazenamento das amostras $\quad 45$

8. Resultados e discussão. $\quad 47$

8.1. Programa de temperatura 47

8.2. Curvas de pirólise e atomização 48

8.3. Massa de modificador. $\quad 50$

8.4. Diluente de fator de diluição

8.5. Estudos de calibração

8.6. Influência da concentração de cálcio e cloreto e fósforo 56 
8.7. Limite de detecção e quantificação. 60

8.8. Estudos de exatidão 61

8.9 Estudos preliminares das espécies organoestânicas

Curvas de pirólise e atomização de compostos organestânicos

63

9 Aplicação

68

10 Conclusões

70

11 Referências bibliográficas 


\section{Lista de figuras}

Figura 1- Curvas de temperatura de pirólise $(\bullet)$ e atomização $(\boldsymbol{\square})$, em meio aquoso $(\boldsymbol{\nabla})$ e no leite humano diluído $1+1$ com $\mathrm{HNO}_{3} \quad 0,2 \%$ v/v ( $\bullet$ ), para 2 ng de iSn em $20 \mu \mathrm{L}$ de $\mathrm{HNO}_{3} 0,2 \%$ v/v, na presença de modificador $\left(15 \mu \mathrm{g}\right.$ de $\mathrm{Pd}+10 \mu \mathrm{g} \mathrm{Mg}\left(\mathrm{NO}_{3}\right)_{2}$ em $10 \mu \mathrm{L}$ de solução). $\mathrm{Tp}=1300^{\circ} \mathrm{C}$ e $\mathrm{Ta}=2200^{\circ} \mathrm{C}$

Figura 2 - Pulsos de absorção de iSn sem modificador (a) e com modificador (b). Sinal AA $(\diamond)$, sinal BG (ロ) em leite humano diluído $1+1$ com $\mathrm{HNO}_{3}$ 0,2\% v/v, para ng de iSn em $20 \mu \mathrm{L}$ de $\mathrm{HNO}_{3}$ $0,2 \% \mathrm{v} / \mathrm{v}$.

Figura 3 - Inclinações (valores relativos) obtidas em curvas de adição de analito (iSn) utilizando-se diferentes massas de modificador. Curva Aquosa (ם), curva no leite $(\boldsymbol{\square}) \mathrm{Tp}=1300^{\circ} \mathrm{C} ; \mathrm{Ta}=2200^{\circ} \mathrm{C}$. Amostras diluídas $1+1$, diluente $\mathrm{HNO}_{3}$ 0,2\% (v/v).

Figura 4- Variação do sinal em função do fator de diluição. Volume $($ amostra $)=20 \mu \mathrm{L}$; modificador: $15 \mu \mathrm{g} \mathrm{Pd}+10 \mu \mathrm{g} \mathrm{Mg}\left(\mathrm{NO}_{3}\right)_{2} \mathrm{em}$ $10 \mu \mathrm{L} ; \mathrm{Tp}=1300^{\circ} \mathrm{C} ; \mathrm{Ta}=2200^{\circ} \mathrm{C}$.

Figura 5 - Curvas de adição de analito em amostras de leite de diferentes doadoras, na faixa de 2,5 a $200 \mu \mathrm{g} . \mathrm{L}^{-1}$; Doadora 1 (•), Doadora 2 (๘), Doadora $3(\triangle)$, Doadora $4(\bullet)$, Doadora $5(*)$. Volume de injeção $=$ $20 \mu \mathrm{L}$; modificador: $15 \mu \mathrm{g} \mathrm{Pd}+10 \mu \mathrm{g} \mathrm{Mg}\left(\mathrm{NO}_{3}\right)_{2}$ em $10 \mu \mathrm{L}$; $\mathrm{Tp}=1300^{\circ} \mathrm{C} ; \mathrm{Ta}=2200^{\circ} \mathrm{C}$. Amostras diluídas $1+1$ com diluente, $\mathrm{HNO}_{3}$ $0,2 \%(\mathrm{v} / \mathrm{v})$.

Figura 6 - Curvas de adição de analito em amostras de leite de diferentes doadoras, na faixa de 2,5 a $10 \mu \mathrm{g} . \mathrm{L}^{-1}$; Doadora 1 ( ), Doadora 2 ( $\left.\square\right)$, Doadora $3(\Delta)$, Doadora $4(\bullet)$, Doadora $5(*)$. Volume de injeção $=$ 20 $\mu \mathrm{L}$; modificador: $15 \mu \mathrm{g} \mathrm{Pd}+10 \mu \mathrm{g} \mathrm{Mg}\left(\mathrm{NO}_{3}\right)_{2}$ em $10 \mu \mathrm{L}$; $\mathrm{Tp}=1300^{\circ} \mathrm{C} ; \mathrm{Ta}=2200^{\circ} \mathrm{C}$. Amostras diluídas $1+1 \mathrm{HNO}_{3} 0,2 \% \mathrm{v} / \mathrm{v}$. 
Figura 7 - Influência da concentração de cálcio sobre o sinal analítico de 2 ng de iSn. Volume de injeção, 20uL; modificador: $15 \mu \mathrm{g}$ de Pd e $10 \mu \mathrm{g}$ de $\mathrm{Mg} ; \mathrm{Tp}=1300^{\circ} \mathrm{C} ; \mathrm{Ta}=2200^{\circ} \mathrm{C}$.

Figura 8 - Influência da concentração de cloreto sobre o sinal analítico de 2 ng iSn. Volume de injeção, 20uL; modificador: $15 \mu \mathrm{g}$ de Pd e $10 \mu \mathrm{g}$ de $\mathrm{Mg} ; \mathrm{Tp}=1300^{\circ} \mathrm{C} ; \mathrm{Ta}=2200^{\circ} \mathrm{C}$.

Figura 9 - Influência da concentração de fósforo sobre o sinal analítico de 2 ng iSn. Volume de injeção, 20uL; modificador: $10 \mu \mathrm{g}$ de Pd e $5 \mu \mathrm{g}$ de $\mathrm{Mg} ; \mathrm{Tp}=1300^{\circ} \mathrm{C} ; \mathrm{Ta}=2200^{\circ} \mathrm{C}$.

Figura 10 - Influência da concentração sobre curvas aquosas: sem a adição de fósforo $(\bullet)$, com adição de $150 \mathrm{mg} . \mathrm{L}^{-1}$ de $\mathrm{P}(\boldsymbol{\Delta}) \mathrm{e}$ com adição de $\mathrm{Ca} / \mathrm{P} / \mathrm{Cl}$ (x) de fósforo sobre o sinal analítico de $2 \mathrm{ng}$ iSn. Volume de injeção, 20uL; modificador: $10 \mu \mathrm{g}$ de $\mathrm{Pd}$ e $5 \mu \mathrm{g}$ de $\mathrm{Mg}$; $\mathrm{Tp}=1300^{\circ} \mathrm{C}$; $\mathrm{Ta}=2200^{\circ} \mathrm{C}$.

Figura 11 - Curva de pirólise $(\diamond)$ e atomização $(\boldsymbol{\Delta})$ para 2 ng de estanho na forma de iSn $(\diamond \mathbf{\Delta})$, MMT $(\diamond \Delta)$, MMT + leite $(\diamond \Delta)$, DMT $(\diamond \Delta)$, DMT + leite $(\diamond \boldsymbol{\Delta})$, TMT $(\diamond \Delta)$ e TMT + leite $(\diamond \boldsymbol{\Delta})$, em $\mathrm{HNO}_{3} \quad 0,2 \% \mathrm{v} / \mathrm{v} ; \mathrm{Tp}=1300^{\circ} \mathrm{C}$ e $\mathrm{Ta}=2200^{\circ} \mathrm{C}$. Volume de amostra: 20uL; massa de modificador: $10 \mu \mathrm{g}$ de Pd e $5 \mu \mathrm{g}$ de Mg em 10uL de solução.

Figura 12 - Curva de pirólise $(\diamond)$ e atomização $(\boldsymbol{\Delta})$ para 2 ng de estanho como iSn $(\diamond \mathbf{\Delta})$ e $\operatorname{MBT}(\diamond \Delta)$, MBT + leite $(\diamond \Delta)$, DBT $(\diamond \Delta)$, $\mathrm{DBT}+$ leite $(\boldsymbol{\Delta})$, e TBT $(\boldsymbol{\Delta})$, TBT + leite $(\bullet \boldsymbol{\Delta})$, em $\mathrm{HNO}_{3}$ 0,2\% v/v. Volume de amostra: 20uL; massa de modificador: $10 \mu \mathrm{g}$ de Pd e $5 \mu \mathrm{g}$ de $\mathrm{Mg}$ em $10 \mathrm{uL}$ de solução.

Figura 13: Curvas no leite de estanho inorgânico $(\diamond), \operatorname{MBT}(\star), \operatorname{DBT}(\Delta)$ e $\mathrm{TBT}(\bullet) . \mathrm{Tp}=1300^{\circ} \mathrm{C}$ e $\mathrm{Ta}=2200^{\circ} \mathrm{C}$. . Volume de amostra: 20uL; massa de modificador: $10 \mu \mathrm{g}$ de $\mathrm{Pd}$ e $5 \mu \mathrm{g}$ de $\mathrm{Mg}$ em $10 \mathrm{uL}$ de solução. Diluição: $1+1$, diluente: $\left.\mathrm{HNO}_{3} 0,2 \%(\mathrm{v} / \mathrm{v}) \mathrm{v} / \mathrm{v}\right)$ 


\section{Lista de tabelas}

Tabela 1 - Principais usos dos organocompostos

Tabela 2 - Programa de temperatura para a determinação de estanho em leite

Tabela 3 - Inclinações relativas e coeficientes de correlação de curvas de adição de analito em diferentes amostras de leite, no intervalo de 2,5 à $200 \mu \mathrm{g} . \mathrm{L}^{-1}$

Tabela 4 - Inclinações relativas e coeficientes de correlação de curvas de adição de analito em diferentes amostras de leite, no intervalo de 2,5 à $10 \mu \mathrm{g} . \mathrm{L}^{-1}$

Tabela 5 - Recuperação (\%) de estanho, adicionado como iSn 62

Tabela 6 - Concentração de iSn em amostras de leite humano 


\section{Lista de Abreviaturas}

iSn - Estanho inorgânico

oSn - Estanho orgânico

$\mu$ g.L-1 - Micrograma por litro

mg.kg-1 - miligrama por quilo

ng.L-1 - nanograma por litro

$\mu \mathrm{L}$ - microlitro

$\mu \mathrm{g}$ - micrograma

${ }^{\circ} \mathrm{C}$ - graus Celsius

MMT - Monometilestanho

DMT - dimetilestanho

TMT - tributilestanho

TeMT - tetrametilestanho

MBT - mnobutilestanho

DBT - dibutilestanho

TBT - tributilestanho

TPT - tripropilestanho

MPhT monofenilestanho

$\mathrm{DPhT}$ - difenilestanho

TPhT - trifenilestanho

TeBT - tetrabutilestanho

TcHexT - triciclohexilestanho

MOT - monooctilestanho

DOT - dioctilestanho

TOT - trioctilestanho

TeOT - tetraoctilestanho

PVC - cloreto de polivinila

TGI - trato gastrointestinal

HAT Atividade da acetiltransferase

FPD - Detetor fotométrico de chama 
GC - cromatografia gasosa

MS - espectrômetro de massas

PFPD - detetor fotométrico de chama pulsante

ICP-MS - plasma indutivamente acoplado com detetor por espectrômetro de massas

ICPOES - plasma indutivamente acoplado com detetor de emissão atômica

HPLC - cromatografia líquida de alta eficiência

HG-GC-QFAAS - espectrometria de absorção atômica em célula de quartzo com geração de hidretos e cromatografia gasosa

ETAAS - espectrometria de absorção atômica eletrotérmica 